\title{
Construction of Rehabilitation System of Freestyle Skiing Aerials Sports Injury
}

\author{
Ningning Zhao, Jian Zhang* \\ Hebei Institute of Sports Science, Shijiazhuang, Hebei 050000, China \\ ${ }^{*}$ Corresponding author
}

Keywords: Freestyle skiing aerial skills, Causes of injury during exercise, To construct a rehabilitation system for injuries

\begin{abstract}
The freestyle skiing Aerials are very stimulating, beautiful and high-tech in sports, which good value for viewing. Therefore, there is a relatively high risk in sports also, and the injury probability in sports of athletes is much higher than that in others. By a variety of investigations and studies, it's found that athletes' injuries are multiple, and most of the injuries are concentrated in the waist, knee, shoulder, ankle and other parts. The author made a detailed analysis on China's freestyle skiing athletes injury situation in this paper. It is hoped that the paper's summary and analysis can provide better methods and suggestions to prevent sports injuries China's freestyle skiing, and effectively reduce the occurrence of injury during exercise, aiming at the injured athletes in sports to set up a healthy body rehabilitation system, it is helpful to promote the higher and farther development of my country's freestyle skiing aerials.
\end{abstract}

\section{Introduction}

\subsection{History and Development of Freestyle Skiing Aerials}

Freestyle skiing aerials, snow skills and snow ballet are the main items of freestyle skiing together. In the 20's last century, American performed a perfect somersault on the snow, which is the rudiment of freestyle skiing. Thirty years later, the Swiss completed a somersault in the slider. Freestyle skiing aerials is very similar to gymnastics and diving. They are all vacated flips in the air, carrying out a lot of difficult moves to complete the entire race. Since 1998,as a performance project in the Winter Olympics, by 1992, aerials are same as snow skills, into the formal events. Because the freestyle skiing aerials fit the human nature's psychology of loving freedom and innovation, it has attracted many viewers since its birth, and with the development of Freestyle Skiing technical conditions, people has continuously innovate more novel skills in freestyle skiing aerials. It's very ornamental, and a visual feast for the audience. For athletes to complete a series of perfect movements in a very short period of time from multiple angles, the athletes will show the advantages and disadvantages of own movement to the audience and judges without reservation. In this process, athletes require to master the balance and control in the air very well. while pursuing stability and self-confidence, also need to accurately hold embrace's movements, showing the aesthetic feeling. Athletes' technical performance of all the movements from the platform to the landing all along the line will determine the athlete's record. The movement of the scoring rule is: every athlete has two trial jump; referees will evaluate the performance of the athletes from the start to the landing. The referee ranks the score of the two trial jump from highest to lowest. On the whole, freestyle skiing aerials are physically demanding

In the late 1980s, Freestyle skiing aerials has been becoming popular in our country. Compared with other countries, Asian's somatotype characteristics is more suitable for freestyle skiing aerials, it is easier to use the light body shape to show the movements to judges and spectators perfectly and freely. The level of the world's freestyle skiing aerials is constantly improving, our country is also learning from the strong ceaselessly, after more than 20 years of continuous efforts, China has now become the leading country in the freestyle skiing aerials, it achieved excellent results on the field repeatedly, the sport is now one of the most skilled in the world. Our country's first winter Olympic Silver medal was won at the 18th Winter Olympic Games in Nagano, Japan in 1988, the twentieth 
winter Olympics in Turin, Italy in 2006, Chinese athletes with a perfect performance to win the Winter Olympics freestyle skiing men's aerials champion, catching the eye of the viewer and the referee, therefore the Chinese freestyle skiing aerials has leaping progress and outstanding achievements, the perfect performance has won the strong attention of North America and some countries with strong skiing skills in Europe.

\subsection{Research Significance}

With the rapid development of economy and sports in the world, the competition of competitive sports also largely represents the competition of comprehensive national strength. Athletes often injured in training is difficult to avoid. Once an athlete is injured before the competition, it will seriously affect his physical and psychological quality about to race, causing athletes cannot play their proper level. Seriously injured people affect daily life, injury is a very serious matter for athletes, will cause enormous negative impact on Athletes' psychology, it needs great attention in the sports training ${ }^{[1]}$. Then, firstly athletes need to ensure a good psychological state in the process of sports training, excessive stress can also lead to problems during training. So the research on the injury of the athletes in the aerials of freestyle skiing has a great effect on the psychological pressure of injury sports and the recovery of good mental state of the athletes. Injured athletes can recover quickly, only if actively cooperating with psychological and physical treatment. Sufficient research on the prevention and countermeasures of injuries in freestyle skiing aerials is conducive to reducing the rate of injuries in sports. More efficient promotion of athletes to achieve better results in the freestyle skiing aerials, improve the skill level of athletes. To stabilize our athletes' level always has been far ahead of the other's excellent state. Thus, we must pay attention to the related research of this sport, in order to develop this sport's competition do a backup guarantee, let freestyle skiing aerials sport has been kept in a good sustainable development state in the world.

\section{Research on Sports Injuries of Athletes}

\subsection{Causes of Relevant Athletes’ Injury in Freestyle Skiing Aerials of Inquiry}

According to some researches on athlete's injury in excellent Freestyle Skiing Aerials, the data shows that athletes' injuries are fairly serious of the China Freestyle Skiing Aerials in sports. Athletes are injuries in the knee, ankle injury parts and the waist commonly, and usually athlete's injury is mainly chronic injury, a little of athletes happens acute injury also, usually, acute injuries are caused by the athlete's poor quality, the incomplete preparation team before training, or didn't comprehend technical essentials.

Many scholars' research proves that the athletes' psychological quality is important factor of related to the athletes in sports injury incident happened. This paper makes a profound discussion on the personality characteristics, psychological factors, and psychological preparation before sports and whether unpleasant events happen in athletes' life. The study found that the main objectives for improve the awareness of self-protection and to minimize and avoid injuries in sports are as follows: the athletes' mentality factors, races pitch and climate factors in the game,the athletes' psychological factors ,the athlete needs to face and solve the unexpected situation in the competition, athletes' own skills and whether the athletes wear good protective gear and other factors.

\subsection{Research on Prevention and Protection of Injuries during Sports}

Athletes were injured in the freestyle skiing aerials movement that athletes need to deal with the matter immediately, many athletes missed the championship because they failed to properly handle the injury when they were injured, and more serious are the injuries that lead to early retirement ${ }^{[2]}$. In our country, there are eight good-level freestyle skiing women's athletes for injuring seriously and cannot normally enter contest, leading more than three people to leave the team, which is a great pity for the athletes' career. Therefore, athletes should pay special attention to warm up before exercise, wearing protective gear and goggles, athletes need to check the suitability of the familiar sports venues before motion, exactly choose skiing equipment and so on, it's important for athletes. 
Through the analysis of skiing athletes are particularly prone to injury data, getting some methods of the necessary preventive and emergency measures for athletes in skiing, Athletes in accept the training and competition for oneself body state or sport- technical level of instability and the interfering psychological factors of some social problems in life, occurring a variety of injury conditions in sports, these injuries include acute and chronic injuries ${ }^{[3]}$. To avoid and solve these movements of all kinds of damage, Athletes should not only improve their physical quality and to feed their sports' level and skills. More importantly, they need to adjust their training and competition to maintain a good mental state. They must learn to manage their emotions, be able to stabilize their emotions at the critical moment, learn to properly relieve the psychological pressure. So as to improve own sports skills and level, and avoid unnecessary physical injury in sports ${ }^{[5]}$.

\subsection{Aiming At Freestyle Skiing Aerial Skills of Sports Injuries Survey Are Analyzed}

There are two categories of athletes in the survey, national team athletes and national youth athletes. The national team had an injury rate of 96.2 percent in the freestyle aerials, and the uninjured rate is less than 4 percent, the national youth athletes' injury rate is 87.6 percent in freestyle skiing aerials, and the uninjured rate was 12.3 percent. Through the above survey shows, in motion, the rate of injury is very high in freestyle skiing aerials' athletes, almost all athletes have the possibility of injury in this sport, thirteen of the 16 national athletes visited were injured, only three were uninjured, injury rates as high as 81.2 percent. Among the 16 national youth team athletes visited, 11 were injured, only 5 people not injured, the number of injury rate was 68.7 percent. Therefore, it is enough to show that the injury is a very common event in sports of Chinese freestyle skiing aerials, movement of the damage rate is very high, injury situation is more serious.

According to the author's investigation result analysis, In our country's freestyle skiing aerials, whether adult athletes and young players' injured area mainly concentrated in the ankle, the waist and knees, among the national team athletes injured, 26 of them suffered waist injuries, accounting for 81.25 percent of the total number, 18 people suffered ankle joint injuries, accounting for 56.25 percent of the total number, the number of knee joint injuries was 29, including 8 retired athletes, accounting for 90.62 percent of the total number. Among the youth injured athletes of the national team, 9 players suffered critical ankle injuries, accounting for 56.25 percent of the total, the waist injured athletes have 17 persons, accounting for 53.12 percent of the total, and the number of knee joint injuries was 15, accounting for 93.75 percent of the total. Integrated the survey shows that the freestyle skiing aerial movement skills in the knee, waist, ankle, three parts are the most vulnerable to damage. In addition, there are also some other more vulnerable parts, such as cervical spine injury, among the adult athletes of the national team, there were 5 cervical spine injuries, accounting for 31.25 percent of the total number, 3 athletes of national youth team suffered cervical spine injuries in sports, accounting for 18.75 percent of the total number. These athletes injuring in the sports must build a good rehabilitation training system, in order to better help athletes recover good physical and mental health, for China's freestyle skiing aerial skills to increase the elegant demeanor.

\section{To Cultivate Training Programs for Injury Awareness and Rehabilitation of Athletes}

\subsection{Training Athletes' Self-Awareness of Injury}

Whether serious or lighter athletic injuries in sport is a blow for the athletes, so it is important for athletes to be aware of their condition and under the guidance of doctors and professional coaches to make the necessary rehabilitation training. Many scientific practices have proved that athletes are much more sensitive to own injuries than others ${ }^{[7]}$. Athletes correct knowledge and understanding of the illness is helpful to prevent the occurrence of sports injuries. If athletes cannot realize their condition correctly, and don't aim at the damage of the body to make the correct rehabilitation training, it's likely to increase the relapse of illness, caused more serious, making athletes greater psychological pressure, therefore, we'll let athletes right to realize their own illness firstly, control body's recovery, according to the situation of the injury, make a suitable rehabilitation training 
system.

\subsection{Strength Training for Athletes during Recovery}

Strength training is focused on the waist and leg muscles, especially the rear muscle strength training is particularly important, static training is recommended when athletes are injured seriously, this period is a total ban on dynamic strength exercise. With the recovery of the movement cloud in the static training, the dynamic training is added gradually, joining the performance practice if there is pain and uncomfortable will immediately cease performance training, and strength training will not resume until the injury has healed.

\subsection{Athletes Take a Flexible Approach to Training}

Athletes should try to stretch as much as possible during injury, enough stretching is good for keeping the body flexible ${ }^{[6]}$. Athletes can first take jogging when you want to stretch the muscles to adapt as soon as possible, according to the fitness of the body in jogging, it is appropriate to change gears, even you can add jumping as you run, or jogging and playing football to conduct rehabilitation training, it can effectively improve the athlete's physical flexibility of the recovery, stretching exercises in training can not only maintain the physical flexibility of athletes, but also help athletes properly relieve body fatigue during training ${ }^{[9]}$.

\subsection{Bullet Net Training Program}

Bullet net training is one of the most popular rehabilitation training in injury training, especially in the process of athletes perceive their own body's recovery and reconstruction, the effect of vertical jump and back spring training using the method of bullet net training is very significant ${ }^{[8]}$. According to their own recovery of proper air over in practice, sport cloud in practice need to wear protective equipment, avoid the secondary damage in training.

\subsection{Image Training Establishes Awareness of Aerial Skill Movements}

Some athletic injuries are chronic and require a long period of training to recover, for athletes of this kind,not only to recover from good physical training at the level of manuscript, also build image training for athletes, the biggest obstacle for athletes with this type of injury is that they are suitable for static exercises in physical recovery training and cannot do normal aerial exercises. Therefore, it is necessary to produce aerial skills in the athletes' mind through image training,repeat training can always remind athletes memory for air action skills, video training should be done every day, and last half an hour each day. Through image to train athletes, even if they can't practice aerials for a long time, also will keep aerials in mind and keep the air skills at a high level.

\section{Typical Rehabilitation Cases and Rehabilitation Plans of Athletes Injured}

From 1999 to 2006, there were 19 athletes in China's national freestyle skiing aerials team, athlete's knee joint twists and ruptures of the anterior cruciate ligament when landing after completing freestyle skiing aerials. There were 5 cases of ligament injury in male athletes, 14 cases in female athletes, 11 cases in left knee injury and 9 cases in right knee injury, including 1 case in both knees. Athletes begin a rehabilitation program the day after surgery. 12 cases were randomly divided into control group, there are 2 cases male athletes, 10 female athletes, 7 had left knee injury and 5 had right knee injury, all damage athletes after special rehabilitation training were not stretch/knee functional disorder.

\subsection{A Classic Case of Lumbar Disc Herniation}

Athletes were unable to train because of a herniated lumbar disc, during the treatment of lumbar disc herniation, continually make a targeted resume training, only one-month time to control the athlete's condition ${ }^{[4]}$, so that athletes began the normal rehabilitation training smoothly. The athlete started pool training in June 2000 and won the first prize in Japan's diving competition in October last year. In January of next year, he took part in the world cup in the US and won fourth place. 
Hitherto unknown breakthrough has been made in the international competition of men's snow sports.

\subsection{Special Rehabilitation Plan for Injured Athletes}

The special training disorders of national athletes have two aspects: clinical rehabilitation and sports rehabilitation. The duration of clinical rehabilitation is controlled according to the recovery of the athlete himself, the athlete's rehabilitation program is conducted by professional doctors and therapists to determine the injury status of the athlete, one by one, the intensity and amount of exercise should be done several times a day according to the endurance of the athlete.

\subsection{Athletes' Rehabilitation Training Plan in Stages}

Around a week after the treatment, athletes need to protect the operative site, reduce postoperative pain and swelling tissue, during this period, it is necessary to maintain proper joint and muscle activity to ensure that the muscles are also strong during the injury. In this predominantly protection period, mainly static exercise, proper to the activities of the joint. An adjustable knee brace should be worn immediately after anterior cruciate ligament reconstruction for joint extension and fixation.

Early post-operative rehabilitation in addition to the injured joint training, other adjacent joints also require early recovery activities, athletes should use ice compress for less than half an hour immediately after each rehabilitation training. Early rehabilitation need to slowly increase the small load endurance exercise training, muscle contractions such as the tether, quadriceps, and adductor bone maintain normal shut-off activity and basic muscle strength exercises. Select the low-load exercise, each group 20 times, each group interval rest 30 seconds, continuous practice two to three groups. Generally, three days after surgery, you can add some weight to your balance exercises.

The early stage of recovery is usually 15 days, when pain and tissue swelling are minimized. Athletes need to gradually restore normal joint activity and increase muscle strength and intensity training. At this stage, the athletes' rehabilitation training is of moderate protection. The focus of the training is to strengthen the muscle weight bearing and balance rest, and to strengthen the active stretching and playing of the knee while maintaining the normal endurance. The Angle of joint movement is from 0 degrees to 140 degrees. When the athlete walks in a normal gait, both lower limb strength should be trained at the same time. The correct training method for the injured is to restore the same muscle strength before the injury.

After the athletes' bodies return to the normal state of motor function and reach the standard through the test of various indicators, they can maintain rehabilitation training for two months under the guidance of rehabilitation doctors, to change the state of motion from a basic motion to a technical motion. The training in this period is mainly strength training. Select the exercise with a larger load, do it 20 times for each group, keep doing three or four groups of exercise in a row, and Take a break for one minute at a time and repeat until tired.

Athletes rehabilitation in the late stage of movement function of intensive training, be outside of the normal activity of joints need to keep muscle strength reaches a certain intensity of training and athletes began to recover injured before the normal training, strengthen the comprehensive recovery of exercise injury, emphatically strengthened the power of each joint and muscle, ligament and flexibility training, establish a good coordinate stability of each part. Gradually increase the athlete's lower limb exercise, such as running, sprinting, stopping, spinning, jumping, skiing and other exercises under general training. When the athlete's body is fully recovered, he/she should carry out the special training of skiing skills to exercise the coordination of the body and the control ability of the body, and train the muscle strength and joint flexibility of the athlete by jumping and flying.

\section{Make Rehabilitation Plan for Athletes Returning to the Competition}

\subsection{Athletes Train Intensively Before Returning to the Field}

All aspects of the test are generally performed after athletes have undergone surgery and four 
months of rehabilitation training. After passing the test, the athlete can begin the rehabilitation training to return to the competition and make full preparation for the competition. The main objective of this stage of training is to new-found the skiing skills of the athlete before the injury. At this time the movement has entered the intensive training stage, the training cycle in about six to eight weeks' time. Athletes during training to maintain the normal muscle strength, according to the special requirements of the special training for high strength of special training, at this time, the intensity and skills of the sport will be improved to the maximum extent of the athlete's ability due to the individual situation, according to the actual capacity of athletes appropriate adjust the intensity and the difficulty of movement skills ${ }^{[10]}$. Do three to four sets of exercise for each item, ten to twelve times for each group, take a one-minute rest interval for each group, and repeat the exercise until you feel tired. During training, if the athlete's ligament tissue reconstruction is not strong enough, the appropriate amount should be paid attention to when practicing, exercise intensity needs to use a gradual mode, remember not to blindly risk training for the progress of training, when strengthening training, all aspects such as muscle, joint, ligament have reached motion standard only then can undertake intensity training.

\subsection{Skills Training and Mental Health Reconstruction of Athletes Before Competition}

Athletes before the competition has to be the corresponding skills training, not only need to determine the test all aspects of physical and technical level, more important is mental health for the reconstruction of the athletes return to the team. The psychological rehabilitation of athletes is an important part in the rehabilitation of sports injuries,the injury of far mobilization will have a great impact on the whole team, and the physical injury of national team players is under great psychological pressure. Therefore, the psychological rehabilitation of athletes in the process of rehabilitation is attracting more and more attention. Most athletes have more or less psychological shadow and pressure after injury. Such negative emotions are the most serious hindrance in the rehabilitation process of athletes, affecting the confidence of physical recovery of athletes, and extending the normal rehabilitation period of athletes. During the recovery period, athletes need to adjust their mentality. They need the understanding and support of teammates, as well as the trust and encouragement of coaches, so that they can make a decision to return to the competition.

According to the past injury history of athletes, some athletes recover faster, and some of them are injured repeatedly in the same part. Then the psychological pressure on the athletes will be greater, which will hurt the confidence of the athletes to recover. So athletes need careful guidance from coaches and constant encouragement and support from teammates after the treatment period. Coaches and rehabilitation therapists play a vital role in rebuilding confidence in the athlete's return to the field. In order to ensure the safety of athletes and make a reasonable rehabilitation plan for athletes, the rehabilitation master not only needs the super technical level of the rehabilitation master, but also needs the extraordinary patience of the rehabilitation master, especially when the athletes need long-term rehabilitation therapy period. During this period, athletes need to feel that they are getting closer to the team. The coach keeps close contact with the athletes during the recovery period, so as to create a warm, encouraged and caring environment for the athletes to conduct rehabilitation training. It is beneficial to the physical and mental rehabilitation of athletes to help athletes return to the competition as soon as possible.

\section{Conclusion}

Now the world's sports level gradually increase improving, winter sports around the world are also developing rapidly. Such as today's popular winter freestyle skiing aerials, the movement was born in the United States in 1960, snow sports were introduced into China in the 1990s, after a few short generations of constant groping practice, the aerials of freestyle skiing have become the dominant traditional skiing events in China. As early as the 2006 Olympic Games in Italy, China achieved a historic good result, among which there are two medal athletes: gold medal Han Xiaopeng, silver medal Li Nina. The injury and rehabilitation system of training and mobilization is particularly important if the sport is to continue to develop and move forward. Athletes need timely 
treatment after injury, pay attention to rest and more need professional rehabilitation training, during the period of professional understanding to give athletes the correct guidance and help, it is difficult for athletes to objectively judge their injuries in their mental state after injury, so professionals should not only make professional rehabilitation plans for injured athletes. In addition, the process of return need special pre-match psychological and physical rehabilitation intensive training. Long-term recovery from chronic injuries requires medical staff and coaches to help athletes work together to overcome negative attitudes and rebuild their determination to return to the field.

\section{References}

[1] Guangzhou Li. The psychological cause of sports injury and psychological intervention measures. Journal of Chongqing Three Gorges University. no.3, PP. 155-157. 2009.

[2] G. Liu, X. Ju on sports Injuries of Chinese elite freestyle skiing aerials athletes. Journal of Harbin Institute of Physical Education. no.6, pp. 155-157, 2008.

[3] Y. Zhang, Y, Zhao, M. Li. Investigation and analysis of sports injuries of Chinese freestyle skiing snowboarding skiers. Journal of Shenyang Physical Education University. no.4, pp. 12-14, 2008.

[4] D. Ma, H. Liu, H. Wei. Investigation and Analysis of sports Injuries of ski enthusiasts in Heilongjiang Province. Journal of Harbin Institute of Physical Education. no.2, pp. 13-15. 2008.

[5] J. Ge, X. Chen, X. Gong, D. Zhang. Fitness function of mass Skiing and prevention of sports Injury. Sports Science and Technology Literature Bulletin. no.10, pp.7-8+26. 2007.

[6] W. Chen, X. Tian. Prevention and treatment of skiers' sports injuries. Ice and Snow Sports. no.2, pp.57-59. 2007.

[7] X. Lin, Colisheng. Observation on therapeutic effect of meniscus injury in freestyle skiing aerials. Ice sports. no.5, 2006.

[8] Z. Wu. Research on the Safety of Freestyle skiing in China. Journal of Shenyang Institute of Physical Education. no.2, pp. 78-80. 2006.

[9] D. Ji, M. Dong. Specialized exercises to prevent injury in freestyle skiing aerials. Ice and snow Sports. no.5, 19+33. 2005.

[10] C. Yang, G. Liu. Type of four seasons freestyle skiing aerial skills training ground research. Ice and snow sports. no.4, pp. 48-50. 2004. 